\title{
Descriptions of four new species of Chrysotimus Loew from Tibet (Diptera: Dolichopodidae)
}

\author{
Mengqing Wang \& Ding Yang*
}

Wang, M. \& Yang, D. 2006: Descriptions of four new species of Chrysotimus Loew from Tibet (Diptera: Dolichopodidae). — Entomol. Fennica 17: 98-104.

The occurrence of the genus Chrysotimus Loew, 1857 in Tibet is confirmed for the first time. The following four species are described as new to science: Chrysotimus bifurcatus sp. n., C. grandis sp. n., C. lii sp. n. and C. linzhiensis sp. n. A key is given to distinguish the Chinese species of the genus.

M. Wang and D. Yang (correspondent author), Department of Entomology, China Agricultural University, Beijing 100094, China

Received 26 January 2005, accepted 15 July 2005

\section{Introduction}

The genus Chrysotimus Loew, 1857 belongs to the subfamily Peloropeodinae. The genus is generally characterized by the yellow or yellowish hairs and bristles, small first flagellomere, hind tarsomere 1 in most males bearing several short erect black ventral bristles at base, and mid tarsomere 1 in male at least as long as tarsomeres 2-4. 60 species were known to occur in the Holarctic and Oriental Realms. Previously, there were 8 species known from the Palaearctic (Negrobov 1991) and 2 species from the Oriental Realms (Becker 1922, Dyte 1975). So far 25 species have been known from China (Parent 1944, Wang et al. 2005, Yang 2001, Yang \& Saigusa 2001a, 2001b, 2005, Zhang et al. 2003).

Guzeriplia Negrobov, 1968 embodies the characters of Chrysotimus Loew in the head and thorax with yellow hairs and bristles and biseriate acr. However, in Guzeriplia, the hypopygium is large (almost as long as abdomen), bearing the long surstylus and cercus. Bickel (2004) noted that it should be within the range of variation of the latter, probably as a synonymy.
In this paper, we describe four species new to science, all found from Tibet, an area lying in the Southwest of China, on the northern side of Himalayas, and bordered with Pakistan, India, Burma, Nepal, and Bhutan. The area belongs to the Palaearctic and Oriental Realms. Perhaps the high-altitude climate is responsible for the restricted occurrence of these four species. We also provide a key to Chrysotimus species males from China.

\section{Material and methods}

The specimens for this study were collected by Fasheng Li (Beijing) from Tibet in 1978 by sweep netting, and are deposited in the Entomological Museum of China Agricultural University (CAU), Beijing. All material is preserved in 75\% alcohol.

Abbreviations are as follows: acr $=$ acrostichal, ad $=$ anterodorsal, av $=$ anteroventral, $\mathrm{dc}=$ dorsocentral, LI = fore leg, LII = mid leg, LIII = hind leg, oc = ocellar, $\mathrm{pd}=$ postero-dorsal, $\mathrm{ph}=$ posthumeral, $\mathrm{pv}=$ posteroventral, $\mathrm{v}=$ ventral. 


\section{Key to species of Chrysotimus Loew from China (males)}

1. Hind tarsomere 1 at most with sparse black bristles at base 2 Hind tarsomere 1 with bundle(s) of black ventral bristles at base

8

2. Hind tarsomere 1 without black ventral bristles at base (not known in grandis) 3 Hind tarsomere 1 with black ventral bristles at base

3. Cercus divided into outer and inner lobes 4 Cercus single. China.

sinensis Parent, 1944

4. Acr absent; mid tarsomere 1 shorter than the tarsomeres 2-5; epandrium with nearly quadrate lateral process. Guangxi guangxiensis Yang \& Saigusa, 2001

Six to seven irregularly paired acr; mid tarsomere 1 longer than the tarsomeres 2-5; epandrium with long hook-like lateral process. Tibet.

grandis sp. n.

5. Antenna with scape and pedicel yellow. Zhejiang basiflavus Yang, 2001

Antenna wholly black.

6. Five dc; acr absent. Zhejiang

Six dc; acr present

apicicurvatus Yang, 2001

7. Four to five irregularly paired acr; hind tarsomere 1 with 6 sparse black ventral bristles on basal 1/4. Guangdong

acutatus Wang, Yang \& Grootaert, 2005

Nine to ten irregularly paired acr; hind tarsomere 1 with 2 sparse black ventral bristles at base. Ningxia

ningxianus Wang, Yang \& Grootaert, 2005

8. Four or five dc; acr absent

Six dc; acr present

9. Arista dorsal; hypandrium with broad lateral process. Zhejiang

dorsalis Yang, 2001

Arista sub-apical; hypandrium with thin finger-like lateral process

10. Hind tarsomere 1 with 3-4 short black spinelike ventral bristles at base. Beijing songshanus Wang, Yang

\& Grootaert, 2005

Hind tarsomere 1 with about (or more than) 10 black ventral bristles at base

11. Hairs and bristles on thorax yellow
Hairs and bristles on thorax brownish or brown 13

12. Fore tarsomere 1 with row of about $10 \mathrm{v}$; hind tarsomere 1 with 22 short black ventral bristles on basal $1 / 4$; surstylus basally without inner process. Gansu

chikuni Wang, Yang \& Grootaert, 2005

Fore tarsomere 1 without row of $\mathrm{v}$; hind tarsomere 1 with less than 20 black ventral bristles on basal 1/4; surstylus basally with inner process. Hubei, Shaanxi, Yunnan

shennongjianus Yang \& Saigusa, 2001

13. Hind tarsomere 1 with about 12 short black ventral bristles; surstylus not furcated apically. Yunnan

bispinus Yang \& Saigusa, 2001 Hind tarsomere 1 with 15-16 short black ventral bristles; surstylus furcated apically. Guangxi

xuae Wang, Yang \& Grootaert, 2005

14. Acr 2-4 pairs

Acr more than 5 pairs

22

15. Hind tarsomere 1 with group of $8-12$ black basal bristles at base (which are somewhat sparse), but without distinct $\mathrm{v}$.

16 Hind tarsomere 1 with 1 (or 2) bundles of black basal ventral bristles, and row of $7-8 \mathrm{pv}$

16. CuAx ratio about 0.2 ; lateral process on epandrium not concave near middle $\quad 17$ $\mathrm{CuAx}$ ratio 0.35 ; lateral process on epandrium concave near middle. Yunnan yunlonganus Yang \& Saigusa, 2001

17. First flagellomere as long as wide; hind tibia without distinct $\mathrm{v}$. Tibet lii sp. n. First flagellomere about 1.5 times wider than long; hind tibia with 2 pv. Tibet

linzhiensis sp. n.

18. R4+5 and M parallel apically; hind tarsomere 1 with bundle of 4-5 black ventral bristles at base

R4+5 and M slightly convergent apically; hind tarsomere 1 with 2 bundles of 3-4 black ventral bristles at base. Shaanxi

bifascia Yang \& Saigusa, 2005

19. Hairs and bristles on thorax yellow or pale; surstylus on epandrium not furcated apically

Hairs and bristles on thorax brown; surstylus on epandrium furcated apically. Guangxi 
sanjiangyuanus Wang, Yang \& Grootaert, 2005

20. Fore tarsomere 1 without rowed v; hind tarsomere 1 with 4-5 black ventral bristles at base

Fore tarsomere 1 with row of 5-6 v; hind tarsomere 1 with 8 black ventral bristles at base. Guangdong

guangdongensis Wang, Yang \& Grootaert, 2005

21. Hind femur with row of ad and pd; cercus long and narrow. Beijing

$$
\text { xiaolongmensis Zhang, Yang }
$$

\& Grootaert, 2003

Hind femur without distinctly $d$; cercus round. Shaanxi

unifascia Yang \& Saigusa, 2005

22. Hind tarsomere 1 with row of about 10 pv. 23 Hind tarsomere 1 without distinct $v \quad 26$

23. Hind tarsomere 1 with 10-12 black ventral basal bristles; mid tibia without distinct $v 24$ Hind tarsomere 1 with about 20 black ventral basal bristles; mid tibia with 1 pv.

24. First flagellomere somewhat round, 2.0 times wider than long; cercus not furcated, dorsal lobe on surstylus thick and straight. Shaanxi qinlingensis Yang \& Saigusa, 2005

First flagellomere subtriangular, about as long as wide; cercus bifurcated, dorsal lobe on surstylus thin and curved. Tibet bifurcatus sp. n.

25. Fore and mid tarsomere 1 without distinct v; epandrium basally with short process. Shaanxi setosus Yang \& Saigusa, 2005 Fore and mid tarsomere 1 each with row of 5$6 \mathrm{v}$; epandrium basally with long broad process. Guangdong

xiaohuangshanus Wang, Yang \& Grootaert, 2005

26. Hind tarsomere 1 with 1 bundle of short black ventral bristles; surstylus rather wide and thick; hypandrium with small apical incision

Hind tarsomere 1 with 2-3 bundles of 14-15 black ventral bristles on basal 1/6 (which contains 1-2 small tight bundles and 1 large loose bundle); surstylus narrow finger-like; hypandrium without apical incision. Yunnan digitatus Yang \& Saigusa, 2001

27. Hairs and bristles on thorax pale or yellow 28 Hairs and bristles on thorax dark brown; hind tarsomere 1 with 15-16 black ventral bristles. Yunnan pingbianus Yang \& Saigusa, 2001

28. Hind tarsomere 1 with 12 short black ventral bristles; 8 irregularly paired acr; cercus with round apex; palpus dark brown. Yunnan. incisus Yang \& Saigusa, 2001

Hind tarsomere 1 with 7-8 short black ventral bristles; 6 irregularly paired acr; cercus with acute apex; palpus yellow. Yunnan lijianganus Yang \& Saigusa, 2001

\section{Descriptions of new species}

\section{Chrysotimus bifurcatus sp. n. (Fig. 1)}

Material examined. Holotype $\widehat{\jmath}$ : China: Tibet, Bomi, Jieda, 3050m a.s.1., 16.VII.1978, leg. F. Li (Beijing).

Diagnosis. All coxae yellow. 6-7 irregularly paired acr short and hair-like. Hind tarsomere 1 with group of 10-12 short black ventral bristles on basal $1 / 5$, and row of $8-9$ pv. Cercus bifurcated.

Description. Male. Head metallic green with

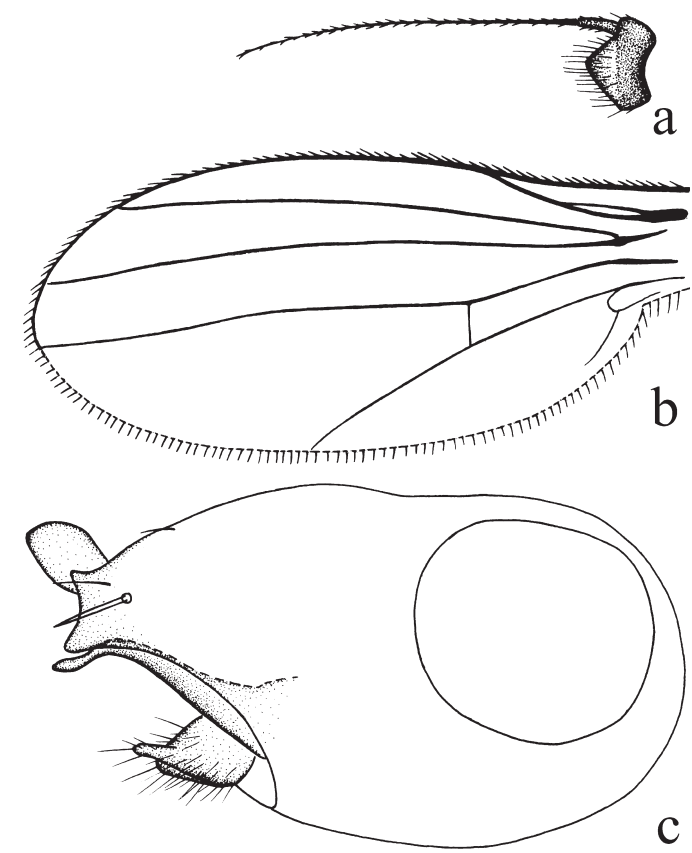

Fig. 1. Chrysotimus bifurcatus sp. n., male. a. First flagellomere, lateral view. b. Wing. c. Genitalia, lateral view. 
pale gray pollen. Hairs and bristles on head yellow. Ocellar tubercle weak, with 2 very long oc and 2 very short posterior hairs. Antenna brown; pedicel with circle of brown apical hairs; first flagellomere subtriangular (Fig. 1a), about 0.6 times as long as wide; arista dorsal, brown, with very short pubescence. Proboscis blackish, with brownish hairs; palpus yellow, with yellow hairs and 2 yellow apical bristles.

Thorax metallic green with pale gray pollen, mesonotum and scutellum brilliant. Hairs and bristles on thorax yellow; 6 strong dc, 6-7 irregularly paired acr, short and hair-like; scutellum with 2 pairs of bristles. Propleuron with 1 yellowish hair and 1 yellowish bristle on lower portion.

Legs yellow, all 5th tarsomeres brown. Hairs and bristles on legs yellow; coxae with yellowish hairs and bristles; fore coxa with 6-8 yellow bristles, mid coxa with 2-4 anterior bristles, hind coxa with 1 yellow outer bristle near middle. Fore femur with 2 av apically, mid and hind femora each with 1 av and 1 pv apically. First tibia with row of short pd, apically with 3 short bristles; mid tibia with 2 ad and 2-3 pd, apically with 4 bristles; hind tibia with 1 ad and 2 pd, apically with 3 bristles. Mid tarsomere 1 with 2-3 v, hind tarsomere 1 with group of 10-12 short black ventral bristles on basal $1 / 5$, and row of $8-9$ pv. Relative lengths of tibia and 5 tarsomeres of legs LI $2.8: 1.5: 0.6$ : $0.5: 0.3: 0.4 ;$ LII $3.5: 1.8: 0.7: 0.6: ?:$ ? (the question marks mean broken segments); LIII 3.8 : $1.4: 0.9: 0.6: 0.3: 0.4$.

Wing hyaline (Fig. 1b); veins brownish, R4+5 and $\mathrm{M}$ parallel apically; $\mathrm{CuAx}$ ratio (length of $\mathrm{m}$ $\mathrm{cu} /$ length of distal portion of CuA) 0.24. Squama brownish yellow with pale hairs. Halter yellow.

Abdomen metallic green with pale gray pollen, dorsum brilliant. Hairs and bristles on abdomen yellow.

Male genitalia (Fig. 1c). Epandrium distinctly longer than wide, with round leaf-like lateral process; surstylus slender, with acute apex; cercus somewhat round, bifurcated apically, one branch short and round, another branch long finger-like; hypandrium short and broad.

Size. Body $1.3 \mathrm{~mm}$, wing $1.7 \mathrm{~mm}$.

Female. Unknown.

Remarks. Chrysotimus bifurcatus sp. n. is similar to Chrysotimus qinlingensis Yang \& Saigusa from Qinling in having row of pv and about 10 black basal ventral bristles on hind tarsomere 1, but can be separated from the latter by the epandrium with slender surstylus and bifurcated cercus. In qinlingensis, the surstylus is thick and straight, and the cercus is not furcated (Yang \& Saigusa 2005).

Distribution. Known only from Tibet.

\section{Chrysotimus grandis sp. n. (Fig. 2)}

Material examined. Holotype $\widehat{\jmath}$ : Tibet: Bomi, Jieda, 3700m a.s.1., 12.VIII.1978, leg. F. Li (Beijing).

Diagnosis. Palpus blackish. 6 strong dc, 6-7 irregularly paired acr short and hair-like. Mid tarsomere 1 longer than the total length of corresponding tarsomeres $2-5$. Surstylus of epandrium with large swollen apex.

Description. Male. Head metallic green with pale gray pollen. Hairs and bristles on head yel-
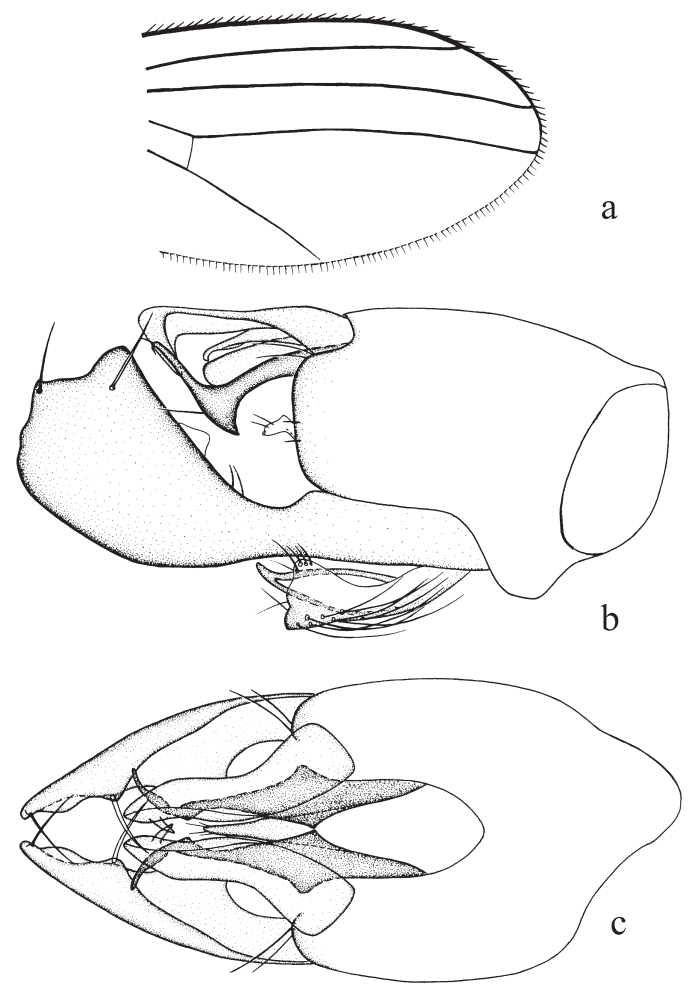

Fig. 2. Chrysotimus grandis sp. n., male. a. Apical half of wing. b. Genitalia, lateral view. c. Genitalia, ventral view. 


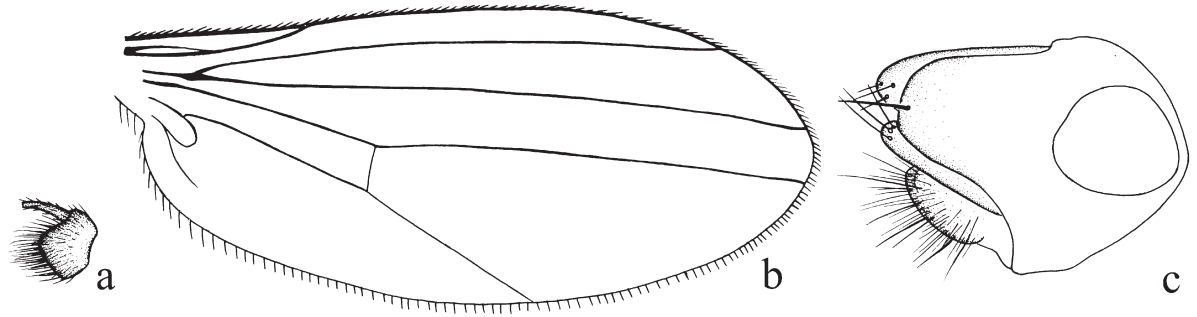

Fig. 3. Chrysotimus lii sp. n., male. a. First flagellomere (not including apical segment of arista), lateral view. b. Wing. c. Genitalia, lateral view.

low. Ocellar tubercle weak, with 2 long oc and 2 short posterior hairs. Antenna broken. Proboscis blackish, with brownish hairs; palpus blackish, with yellow hairs.

Thorax metallic green with pale gray pollen, mesonotum and scutellum brilliant. Hairs and bristles on thorax yellow; 6 strong de, 6-7 irregularly paired acr short and hair-like; scutellum with 2 pairs of bristles. Propleuron with 1 yellowish hair and 1 yellowish bristle on lower portion.

Legs [fore tarsus, hind tibia and tarsus broken] yellow; mid tarsomere 5 brown. Hairs and bristles on legs yellow; coxae with yellowish hairs and bristles; fore coxa with 4-6 yellow bristles, mid coxa with 2-4 anterior bristles, hind coxa with 1 yellow outer bristle near middle. Mid femur with 1 av and $1 \mathrm{pv}$ at apex. Mid tibia with 2 ad and $3 \mathrm{pd}$, apically with 4 bristles. Mid tarsomere 1 longer than the total length of corresponding tarsomeres 2-5. Relative lengths of tibia and 5 tarsomeres of legs LI $3.2:$ ? : ?: ? : ? ? ; LII $4.0: 2.7: 1.0: 0.6: 0.3: 0.5$.

Wing hyaline (Fig. 2a), veins brownish, R4+5 and $\mathrm{M}$ parallel apically; $\mathrm{CuAx}$ ratio 0.2. Squama brownish yellow with pale hairs. Halter yellow.

Abdomen metallic green with pale gray pollen, dorsum brilliant. Hairs and bristles on abdomen yellow.

Male genitalia (Figs 2b-c). Epandrium distinctly longer than wide, somewhat square, with long hook-like lateral process; surstylus with large dorsal lobe, thick base and distinctly swollen apex, and long curved ventral lobe; cercus long and bifurcated, outer lobe with apex wider than base, with several (about 7-8) pale long soft apical hairs, inner lobe long and acute; hypandrium thin and short, symmetrical in ventral view.

Size. Body $1.3 \mathrm{~mm}$, wing $1.6 \mathrm{~mm}$.
Female. Unknown.

Remarks. Chrysotimus grandis sp. n. is somewhat similar to Chrysotimus ningxianus Wang, Yang \& Grootaert from Ningxia in having the long mid tarsomere 1, but can be separated from the latter by the following features: 6-7 irregularly paired acr, mid tarsomere 1 longer than tarsomeres 2-5, and surstylus large with swollen apex; in ningxianus, 9-10 irregularly paired acr, mid tarsomere 1 nearly as long as tarsomeres 2-5, and surstylus slender (Wang et al. 2005).

Distribution. Known only from Tibet.

\section{Chrysotimus lii sp. n. (Fig. 3)}

Material examined. Holotype ${ }^{\top}$ : Tibet: Bomi, Jieda, 3050m a.s.1., 16.VII.1978, leg. F. Li. Paratypes 2 ふึ๋: Tibet: Linzhi, 1.-3.VI.1978, leg. F. Li (Beijing).

Diagnosis. First flagellomere subtriangular, as long as wide. Hind tarsomere 1 with group of 10-11 short black ventral bristles on basal 1/6.

Description. Male. Head metallic green with pale gray pollen. Hairs and bristles on head yellow. Ocellar tubercle weak, with 2 long oc and 2 short posterior hairs. Antenna brown; pedicel with circle of brownish apical hairs; first flagellomere subtriangular (Fig. 3a), as long as wide; arista [apical segment broken] dorsal, brownish, with very short pubescence. Proboscis blackish, with brownish hairs; palpus pale yellow, with yellow hairs and 2 brownish apical bristles.

Thorax metallic green with pale gray pollen, mesonotum and scutellum brilliant, pleuron brown with pale pollen. Hairs and bristles on thorax yellow; 6 strong dc, 3-4 irregularly paired acr short and hair-like; scutellum with 2 pairs of bris- 
tles. Propleuron with 1 yellowish hair and 1 yellowish bristle on lower portion.

Legs yellow, all 5th tarsomeres brown. Hairs and bristles on legs yellow; coxae with yellowish hairs and bristles; fore coxa with 2 yellow bristles, mid coxa with 2-4 anterior bristles, hind coxa with 1 yellow outer bristle at middle. Mid and hind femora each with 1 av and 1 pv at apex. Mid tibia with 2 ad and 2 pd; hind tibia with 2 ad. Hind tarsomere 1 with group of 10-11 short black ventral bristles on basal 1/6. Relative lengths of tibia and 5 tarsomeres of legs LI $3.7: 2.0: 0.9: 0.6$ $: 0.4: 0.5 ;$ LII $4.7: 2.6: 1.0: 0.7: 0.4: 0.4 ;$ LIII 5.0 $: 2.3: 1.1: 0.8: 0.5: 0.5$.

Wing hyaline (Fig. 3b); veins brownish, R4+5 and $\mathrm{M}$ parallel apically; $\mathrm{CuAx}$ ratio 0.22 . Squama brownish yellow with pale hairs. Halter yellow.

Abdomen metallic green with pale gray pollen, dorsum brilliant. Hairs and bristles on abdomen yellow.

Male genitalia (Fig. 3c). Epandrium distinctly longer than wide, with wide lateral process; surstylus with round apex, with 3 long apical bristles; cercus long and rather narrow apically; hypandrium short and broad with round apex.

Size. Body $1.6 \mathrm{~mm}$, wing $2.5 \mathrm{~mm}$.

Female. Unknown.

Remarks. Chrysotimus lii sp. n. is similar to Chrysotimus bifascia Yang \& Saigusa from Qinling in having the pale yellow palpus and brown 5 th tarsomere, but can be separated from the latter by the hind tarsomere 1 with 10-11 black ventral bristles at base and the first flagellomere as long as wide. In bifascia, the hind tarsomere 1 just has 3-4 black ventral bristles, and the first flagellomere is about 0.6 times as long as wide (Yang \& Saigusa 2005).

Distribution. Known only from Tibet.

\section{Chrysotimus linzhiensis sp. n. (Fig. 4)}

Material examined. Holotype $\widehat{\jmath}$ : Tibet: Linzhi, 3050m a.s.1., 3.VI.1978, leg, F. Li (Beijing).

Diagnosis. Palpus pale yellow. Legs yellow with 5th tarsomeres brown. Hind tarsomere 1 with group of 13 short black ventral bristles on basal $1 / 6$.

Description. Male. Head metallic green with pale gray pollen. Hairs and bristles on head yel-

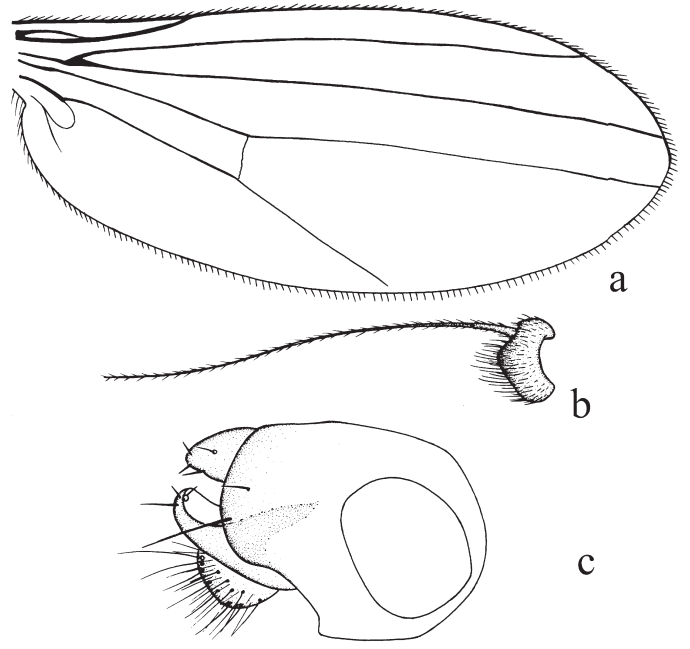

Fig. 4. Chrysotimus linzhiensis sp. n., male. a. Wing. b. First flagellomere, lateral view. c. Genitalia, lateral view.

low. Ocellar tubercle weak, with 2 very long oc and 2 very short posterior hairs. Antenna brown; pedicel with circle of brown apical hairs; first flagellomere rather wide (Fig. 4b), about 1.5 times wider than long; arista dorsal, brown, with short pubescence. Proboscis blackish, with brownish hairs; palpus pale yellow, with yellow hairs and 2 yellow apical bristles.

Thorax metallic green with pale gray pollen, mesonotum and scutellum brilliant, pleuron brown with pale pollen. Hairs and bristles on thorax yellow; 6 strong dc (the first de shorter), 4 irregularly paired acr, short and hair-like; scutellum with 2 pairs of bristles. Propleuron with 1 yellowish hair and 1 yellowish bristle on lower portion.

Legs yellow, all 5th tarsomeres brown. Hairs and bristles on legs yellow; coxae with yellowish hairs and bristles; fore coxa with 4 yellow bristles, mid coxa with 2-4 anterior bristles, hind coxa with 1 yellow outer bristle at middle. Mid and hind femora each with 1 av and 1 pv apically. Mid tibia with 2 ad and $1 \mathrm{pd}$; hind tibia with 1-2 ad, $1 \mathrm{pd}$ and $2 \mathrm{pv}$. Hind tarsomere 1 with group of 13 short black ventral bristles on basal 1/6. Relative lengths of tibia and 5 tarsomeres of legs LI $4.0: 2.1: 1.0: 0.6: 0.4: 0.5 ;$ LII $4.7: 2.7: 1.1: 0.8$ $: 0.4: 0.4$; LIII $4.8: 2.1: ?: ?: ?: ?$.

Wing hyaline (Fig. 4a), veins brownish, R4+5 and $\mathrm{M}$ parallel apically; $\mathrm{CuAx}$ ratio 0.25. Squama 
brownish yellow with pale hairs. Halter yellow.

Abdomen metallic green with pale grey pollen, dorsum brilliant. Hairs and bristles on abdomen yellow.

Male genitalia (Figs. 4c). Epandrium distinctly longer than wide with wide lateral process; surstylus curved downward, round apically, with 3 apical bristles; cercus round with yellow marginal bristles and hairs; hypandrium short and broad.

Size. Body $1.8 \mathrm{~mm}$, wing $2.6 \mathrm{~mm}$.

Female. Unknown.

Remarks. Chrysotimus linzhiensis sp. $\mathrm{n}$. is similar to Chrysotimus yunlonganus Yang \& Saigusa from Yunnan in having the short first flagellomere and 3-4 paired acr, but can be separated from the latter by the hind tarsomere 1 with 13 black ventral bristles at base and curved surstylus. In yunlonganus, the hind tarsomere 1 just has 8 sparse black ventral bristles, and the surstylus is not curved (Yang \& Saigusa 2001b).

Distribution. Known only from Tibet.

Acknowledgements. The authors wish to thank Mr. Fasheng Li (Beijing) for collecting specimens and help in many ways. This study was supported by the National Natural Science Foundation of China (No. 30225009).

\section{References}

Becker, T. 1922: Dipterologische Studien. Dolichopodidae der Indo-Australische Region. — Capita Zoologica 1 (4): 1-247.
Bickel, D. J. 2004: Alishania, a new genus with remarkable female terminalia from Taiwan, with notes on Chrysotimus Loew (Diptera: Dolichopodidae). Bishop Mus. Bull. Ent. 12: 27-34.

Dyte, C. E. 1975: Family Dolichopodidae. - In: Delfinado, M. D. \& Hardy, D. E. (eds.), A catalog of the Diptera of the Oriental region, 2: 212-258. Hawaii University Press, Honolulu. 618 pp.

Loew, H. 1857: Neue Beitr e zur Kenntniss der Dipteren. F fter Beitrag. — Programm K. Realschule Meserit, 1857: 1-6.

Negrobov, O. P. 1991: Family Dolichopodidae. - In: Soós, Á. \& Papp, L. (eds.), Catalogue of Palaearctic Diptera 7: 11-139. Akad iai Kiad Budapest. 291 pp.

Parent, O. 1944: Diptères Dolichopodidae recueillis en Chine de Nord, en Mongolie et en Mandchourie par le P. P. E. Licent. - Rev. Fr. Ent. 10 (4): 121-131.

Wang, M., Yang, D. \& Grootaert, P. 2005: Chrysotimus Loew from China (Diptera: Dolichopodidae). Zootaxa 1003: 1-32.

Yang, D. 2001: Diptera: Dolichopodidae. - In: Wu, H. \& Pan, C. (eds.), Insects of the Tianmushan Mountain: 428-441. Science Press, Beijing. 764 pp. [In Chinese.]

Yang, D. \& Saigusa, T. 2001a: New and little known species of Dolichopodidiae (Diptera) from China (VIII). —Bull. Inst. Royal Sci. Nat. Belg. Ent. 71: 155-164.

Yang, D. \& Saigusa, T. 2001b: New and little known species of Dolichopodidiae (Diptera) from China (IX). Bull. Inst. Royal Sci. Nat. Belg. Ent. 71: 165-188.

Yang, D. \& Saigusa, T. 2005: Diptera: Dolichopodidae. In: Yang, X. (ed.), Insect Fauna of Middle-West Qinling Range and South Mountains of Gansu Province: 740-765. Science Press, Beijing. 1055 pp. [In Chinese.]

Zhang, L., Yang, D. \& Grootaert, P. 2003: New species of Chrysotimus and Hercostomus from Beijing (Diptera: Dolichopodidae). — Bull. Inst. Royal Sci. Nat. Belg. Ent. 73: 189-194. 